\title{
ESSENTIAL OIL COMPOSITION OF INDIGENOUS POPULATIONS OF HYPERICUM PERFORATUM L. FROM SOUTHERN ALBANIA
}

\author{
Nikoll Bardhi ${ }^{1}$, Gjoše Stefkov ${ }^{2}$, Marija Karapandzova² $^{2}$ Ivana Cvetkovikj²*, \\ Svetlana Kulevanova ${ }^{2}$ \\ ${ }^{1}$ Nikoll Bardhi, Department of Plant Production, Faculty of Agriculture and Environment, \\ Agriculture University of Tirana, Kodër-Kamëz, Tirana, Albania \\ ${ }^{2}$ Institute of Pharmacognosy, Faculty of Pharmacy, Ss. Cyril and Methodius University, \\ Majka Tereza 47, 1000 Skopje, R. Macedonia \\ ivanacvetkovikj@ff.ukim.edu.mk, ivanacvetkovikj@gmail.com
}

The aim of this study was to investigate the yield and chemical composition of the essential oil (EO) isolated from over-ground parts of different populations of Hypericum perforatum L. (Hypericaceae) (HP) from southern Albania. The EO yield of 11 specimens of indigenous populations of HP ranged from $2.50 \mathrm{ml} / \mathrm{kg}$ to $11.00 \mathrm{ml} / \mathrm{kg}$. GC/FID/MS analyses of the EOs revealed a total of 126 identified compounds representing $77.35-88.29 \%$ of the oils. Based on the prevalence of principal components, two types of EO were distinguished: pinene-type, which included seven populations with EO rich in $\alpha$-pinene, and caryophyllene-type, which included four populations with EO rich in trans- $(E)$ caryophyllene and caryophyllene oxide. The information obtained can help to assess the potential of the studied Albanian populations for further sustainable wild exploitation to take it into a consideration as a resource of valuable genetic material or for further cultivation and breeding.

Keywords: Saint John's wort; GC/FID/MS; wild populations; types of oil

\section{СОСТАВ НА ЕТЕРИЧНО МАСЛО ОД ДИВОРАСТЕЧКИ ПОПУЛАЦИИ НА HYPERICUM PERFORATUM L. ОД ЈУЖНА АЛБАНИЈА}

Целта на оваа студија беше да се испита приносот и хемискиот состав на етеричните масла (EO) изолирани од надземниот дел на различни популации на растението Hypericum perforatum L. (Нуреricaceae) (НР) од јужна Албанија. Содржината на маслото од 11 диворастечки популации на $\mathrm{HP}$ се движи од 2,50 ml $/ \mathrm{kg}$ до 11,00 ml/kg. Co GC/FID/MS беше извршена анализа на соодветните етерични масла, беа идентификувани вкупно 126 компоненти, што претставува 77,35\% до 88,29\% од вкупната содржина на маслата. Врз основа на застапеноста на главните компоненти, се разликуваат два типа масла: пиненски тип, во кој спаѓаат седум популации чијашто главна индивидуална компонента е $\alpha$-пинен, и кариофиленски тип, во кој спаѓаат четири популации богати co trans-(E)-кариофилен и кариофилен оксид. Добиените резултати можат да придонесат за процена на потенцијалот на албанските диворастечки популации за понатамошна нивна експлоатација, но и нивно користење како вреден извор на генетски материјал или за нивно култивирање и размножување.

Клучни зборови: кантарион; GC/FID/MS; диворастечки популации; типови на етерично масло 


\section{INTRODUCTION}

The Hypericum species are perennial plants distributed predominantly in the temperate regions of the world with approximately 470 species in total [1]. Hypericum perforatum L. (Hypericaceae) (Saint John's wort), the most thoroughly studied taxon of the genus, is well known for its traditional and contemporary use as a medicinal plant, with a complex mixture of secondary metabolites. Due to the large range of secondary metabolites that are active principals of phytopharmaceuticals, Hypericum perforatum (HP) possess a plethora of pharmacological activities including antiviral, antimicrobial, anti-inflammatory, antioxidant, antidepressive, hepatoprotective and anti-tumoral [26]. An infused oil of the flowers (Hyperici oleum), which is prepared by macerating fresh flowers in olive or sunflower oil and exposing the mixture to sunlight for two to three weeks, has a history of traditional use in Europe for the treatment of burns and ulcers [7, 8]. Monographs for the crude drug and extracts of HP, prepared from the aerial flowering parts of the plant, have been fully integrated in the European Pharmacopoeia (since Ph. Eur. 6) [2]. Hyperici oleum was included in the German Commission E and the Swiss Pharmacopoeia monographs [7, 9].

The bioactive compounds found in HP comprise the naphthodianthrone derivatives (hypericin and pseudohypericin), acylated phloroglucinol derivatives (hyperforin and adhyperforin), flavonoids (quercetin, quercitrin, hyperoside, rutin, kaempferol, biapigenin and amentoflavone), phenolic acids (chlorogenic acid) and xanthones (mangiferin) [4, 5]. A number of studies refer to the composition of the essential oil (EO) of HP [10-16]. Typical HP EO constituents for the material collected in southern Europe (France, Italy, Turkey and Serbia) include the monoterpenes $\alpha$ and $\beta$-pinene, limonene and myrcene, the sesquiterpenes trans- $(E)$-caryophyllene and caryophyllene oxide [17-20] and hydrocarbons such as $n$-decane, $\mathrm{C}_{16}$ and $\mathrm{C}_{29}$ alkanes and $\mathrm{C}_{24}, \mathrm{C}_{26}$ and $\mathrm{C}_{28}$-alkanols [21]. Hypericum species are generally classed as essential oil-poor plants (oil yield $<1.00 \%, w / w)[22,23]$. The content of EO in HP is highest during the full-bloom stage versus the pre-bloom or fruiting stage $(0.35 \%$ versus $0.12 \%$ and $0.18 \%$, respectively) [23-25]. Despite the poor content, the steam-distilled EO of HP has recently become available on a broader scale on the market and is largely produced by small companies in Eu- rope (Poland, France), especially in some South East European countries (Serbia, Croatia and Bulgaria) [26]. Despite the abundant literature data about the EO composition of HP growing in southern Europe, to date, there are no data about chemical investigations performed on samples from Albania. Therefore, the aim of this study was to investigate the yield and the chemical composition of the EO isolated from over-ground parts of different populations of HP from southern Albania, estimating the chemical potential of wild exploitation of this plant for HP EO isolation.

\section{EXPERIMENTAL SECTION}

Plant material. Aerial flowering parts of Hypericum perforatum populations from 11 different locations from southern Albania were collected. The plant material was air dried, packed in paper bags and kept in a dark and cold place until analysis. Plant identity was verified and voucher specimens were deposited at the Institute of Pharmacognosy, Faculty of Pharmacy, Skopje, R. Macedonia (Table 1).

Essential oil isolation. The EO was isolated from dried and minced plant material by hydrodistillation in all-glass Clevenger apparatus for 2 hours according to $\mathrm{Ph}$. Eur. The obtained oil was dried with anhydrous $\mathrm{Na}_{2} \mathrm{SO}_{4}$ and dissolved in hexane for further analyses.

Analysis of essential oils' chemical composition. EO samples in hexane (1:1000) were analyzed on Agilent 7890A Gas Chromatography system equipped with FID detector and Agilent 5975C mass spectrometer. For that purpose, HP-5 ms capillary column $(30 \mathrm{~m} \times 0.25 \mathrm{~mm}$, film thickness $0.25 \mu \mathrm{m})$ was used. Analytical conditions were as follows: oven temperature at $60^{\circ} \mathrm{C}(0$ $\mathrm{min}), 3{ }^{\circ} \mathrm{C} / \mathrm{min}$ to $240{ }^{\circ} \mathrm{C}(1 \mathrm{~min})$ and at the end increased to $280{ }^{\circ} \mathrm{C}$ at a rate of $10{ }^{\circ} \mathrm{C} / \mathrm{min}(1 \mathrm{~min})$; helium as carrier gas at a flow rate of $1 \mathrm{ml} / \mathrm{min}$; injector temperature $220^{\circ} \mathrm{C}$ and that of the FID detector $270{ }^{\circ} \mathrm{C}$. One $\mu \mathrm{l}$ of each sample was injected at a split ratio of 1:1. The mass spectrometry conditions were: ionization voltage $70 \mathrm{eV}$, ion source temperature $230{ }^{\circ} \mathrm{C}$, transfer line temperature $280{ }^{\circ} \mathrm{C}$, and mass range from $50-550 \mathrm{Da}$. The MS was operated in scan mode.

Identification of the components. The compounds were identified on the basis of literature [27] and estimated Kovat's (retention) indices that were determined using a mixture of homologous series of normal alkanes (C9-C25) analyzed under Automated Mass Spectral Deconvolution and Iden- 
tification System (AMDIS)' conditions [28]. Confirmation was made by comparing the mass spectra of the components present in the EOs with the reference spectra obtained from Nist, Wiley and Adams mass spectra libraries. Quantification of the EOs components was performed using the normal- ization method of the GC/FID peak areas without any correction factors.

Statistical analysis. The Principal Component Analysis (PCA) based on seven main essential oil constituents was performed using SIMCA 13 [29].

Table 1

Sampling localities and essential oil (EO) yields of 11 Hypericum perforatum L. populations from southern Albania

\begin{tabular}{|c|c|c|c|c|c|c|}
\hline Population & $\begin{array}{l}\text { (Albania) } \\
\text { Town }\end{array}$ & Latitude (N) & Longitude (E) & $\begin{array}{l}\text { Height above } \\
\text { the sea level } \\
\text { (m) }\end{array}$ & $\begin{array}{l}\text { Date of getting } \\
\text { the sample }\end{array}$ & $\begin{array}{c}\text { EO yield } \\
\mathrm{ml} / \mathrm{kg}\end{array}$ \\
\hline HP01 & Kolonja & $40^{\circ} 18^{\prime} 42.75^{\prime \prime}$ & $20^{\circ} 39^{\prime} 55.42 ”$ & 1095 & 12.7.2011 & 5.00 \\
\hline HP02 & Korca & $40^{\circ} 36 ’ 50.90^{\prime \prime}$ & $20^{\circ} 46^{\prime} 39.81 "$ & 875 & 12.7.2011 & 4.00 \\
\hline HP03 & Pogradec & $40^{\circ} 54^{\prime} 01.83^{\prime \prime}$ & $20^{\circ} 39^{\prime} 50.12^{\prime \prime}$ & 742 & 12.7.2011 & 4.00 \\
\hline HP04 & Elbasan & $41^{\circ} 07^{\prime} 01.68^{\prime \prime}$ & $20^{\circ} 05^{\prime} 32.97^{\prime \prime}$ & 284 & 13.7 .2011 & 3.00 \\
\hline HP05 & Tirana & $41^{\circ} 19^{\prime} 57.88^{\prime \prime}$ & $19^{\circ} 49^{\prime} 53.23 ”$ & 163 & 15.7.2011 & 2.50 \\
\hline HP06 & Berat & $50^{\circ} 42^{\prime} 31.19 ”$ & $19^{\circ} 56^{\prime} 37.30^{\prime \prime}$ & 234 & 13.7.2011 & 10.00 \\
\hline HP07 & Lushnje & $40^{\circ} 56^{\prime} 00.08^{\prime \prime}$ & $19^{\circ} 42^{\prime} 00.11^{\prime \prime}$ & 226 & 13.7.2011 & 4.00 \\
\hline HP08 & Fier & $40^{\circ} 43^{\prime} 48.47^{\prime \prime}$ & $19^{\circ} 34^{\prime} 23.41^{\prime \prime}$ & 215 & 13.7.2011 & 11.00 \\
\hline HP09 & Tepelene & $40^{\circ} 18^{\prime} 00.03^{\prime \prime}$ & $20^{\circ} 00 ' 59.96 "$ & 674 & 14.7 .2011 & 4.00 \\
\hline HP10 & Gjirokastra & $40^{\circ} 04^{\prime} 45.11^{\prime \prime}$ & $20^{\circ} 08^{\prime} 53.53^{\prime \prime}$ & 318 & 14.7.2011 & 11.00 \\
\hline HP11 & Saranda & $39^{\circ} 52^{\prime} 36.42^{\prime \prime}$ & $20^{\circ} 00^{\prime} 00.68^{\prime \prime}$ & 243 & 14.7 .2011 & 9.00 \\
\hline
\end{tabular}

\section{RESULTS AND DISCUSSION}

Essential oil yield. The EO yield of 11 specimens of indigenous populations of HP from southern Albania ranged from $2.50 \mathrm{ml} / \mathrm{kg}$ to 11.00 $\mathrm{ml} / \mathrm{kg}(0.25-1.10 \%)$ (Table 1$)$. The highest yield was recorded in two indigenous populations from the regions of Fier and Gjirokastra (HP08 and HP10, respectively) while the lowest was found in the population located in Tirana region (HP05). Differences in the EO content were evident when comparing populations from the vicinity of the Saranda, Gjirokastra, Fier and Berat, which are cities located closer to the Ionic See, to those from Tirana and Lushnje, located more northern, and Podgradec, Kolonja, Korcha and Elbasan, located more eastern. According to literature data, large variation in EO content $(0.04-1.93 \%)$ was also reported for plants from six different localities in Serbia [30]. Additionally, the yields of EOs from HP from Kosovo differed greatly depending on the population and ranged from 0.04 to $0.26 \%$ based on dry weight [31].
EO composition. Data analysis of the EO chemical composition revealed six different classes of components: monoterpene hydrocarbons (MH), oxygen-containing monoterpenes (OM), sesquiterpene hydrocarbons ( $\mathrm{SH})$, oxygencontaining sesquiterpenes (OS), diterpenes (D) and non-terpene components (NT). Generally, $\mathrm{MH}$ and $\mathrm{SH}$ were dominant fractions in all tested samples. In this order, six populations contained EO rich in $\mathrm{SH}$, while the EOs isolated from five other populations showed the MH fraction to be dominant. Additionally, oxygen-containing derivatives were present in lower amounts. Nine of the 11 populations encompassed EO that contained more OS, while only two EO populations had OM as the main fraction. Regarding the content of diterpenes (D), it is important to emphasize that manool is the only diterpene compound that was identified in only one EO sample. Beside terpene compounds, these EOs contained high levels of other non-terpene components (NT) which mainly consisted of aliphatic chains (Table 2). 
T a ble 2

Chemical composition (\%) of the essential oils from 11 Hypericum perfratum L. populations, HP01-HP11

\begin{tabular}{|c|c|c|c|c|c|c|c|c|c|c|c|c|c|c|}
\hline No. & Components & $\mathbf{K I L}^{\text {a) }}$ & $\mathbf{K I E}^{\mathbf{b})}$ & HP01 & HP02 & HP03 & HP04 & HP05 & HP06 & HP07 & HP08 & HP09 & HP10 & HP11 \\
\hline 1 & Cumene & 926 & 929 & 2.67 & 2.66 & 3.71 & 1.78 & - & 0.86 & 0.90 & 0.37 & 0.66 & - & - \\
\hline 2 & $\alpha$-Pinene & 939 & 941 & 13.37 & 14.03 & 12.65 & 2.03 & 20.61 & 7.03 & 29.48 & 1.15 & 15.31 & 36.74 & 26.52 \\
\hline 3 & Camphene & 953 & 957 & 0.09 & - & - & - & - & - & - & - & - & - & - \\
\hline 4 & Propylbenzene & 960 & 964 & 0.10 & 0.06 & 0.11 & 0.10 & - & 0.04 & - & 0.06 & 0.08 & - & - \\
\hline 5 & $\begin{array}{l}\text { 3-Methyl- } \\
\text { nonane }\end{array}$ & 971 & 974 & 0.63 & 0.77 & 0.73 & 0.76 & 1.55 & 0.98 & 1.82 & 0.54 & 1.56 & 2.76 & 2.41 \\
\hline 6 & Sabinene & 976 & 984 & - & - & - & - & - & 0.04 & - & - & - & - & - \\
\hline 7 & $\beta$-Pinene & 980 & 985 & 1.88 & 5.55 & 3.99 & 0.36 & 6.81 & 0.62 & 2.98 & 0.30 & 6.89 & 6.70 & 1.95 \\
\hline 8 & $\beta$-Myrcene & 991 & 996 & 0.23 & 0.33 & 0.33 & 0.15 & 0.93 & 0.31 & 0.90 & 0.10 & 0.66 & 0.39 & 0.60 \\
\hline 9 & $n$-Decane & 999 & 999 & 0.06 & - & - & 0.06 & 0.16 & 0.08 & 0.20 & 0.03 & 0.14 & 0.18 & 0.23 \\
\hline 10 & $\alpha$-Phellandrene & 1005 & 1010 & - & - & - & - & 0.05 & 0.03 & 0.04 & - & - & 0.08 & 0.06 \\
\hline 11 & $\alpha$-Terpinene & 1018 & 1023 & - & - & - & - & 0.07 & & 0.04 & - & - & 0.06 & 0.06 \\
\hline 12 & o-Cymene & 1022 & 1029 & - & 0.05 & 0.05 & 0.06 & 0.17 & 0.12 & 0.16 & - & 0.29 & 0.91 & 0.37 \\
\hline 13 & Limonene & 1024 & 1031 & - & 0.28 & 0.28 & 0.12 & 0.86 & 0.34 & 0.60 & 0.07 & 0.70 & 2.03 & 0.86 \\
\hline 14 & 1,8-Cineole & 1026 & 1034 & - & 0.05 & 0.04 & - & - & - & - & - & - & - & 0.29 \\
\hline 15 & $\beta$-Z-Ocymene & 1032 & 1035 & - & - & - & 0.06 & 0.12 & 0.05 & - & 0.06 & - & - & - \\
\hline 16 & $\beta$-E-Ocymene & 1044 & 1039 & 0.18 & 0.24 & 0.22 & 0.24 & 0.38 & 0.18 & 0.35 & 0.26 & 0.29 & 0.06 & 0.14 \\
\hline 17 & $\gamma$-Terpinene & 1062 & 1069 & - & 0.06 & 0.08 & - & 0.21 & - & 0.15 & - & - & 0.24 & 0.21 \\
\hline 18 & $\begin{array}{l}\text { 2-Methyl- } \\
\text { decane }\end{array}$ & 1064 & 1070 & 1.05 & 1.54 & 1.65 & 1.02 & 2.28 & 1.67 & 2.73 & 0.82 & 2.95 & 3.14 & 2.67 \\
\hline 19 & $\begin{array}{l}\text { cis-Linalol } \\
\text { oxide }\end{array}$ & 1067 & 1073 & 0.13 & 0.12 & - & - & - & 0.09 & - & 0.04 & - & - & - \\
\hline 20 & $\begin{array}{l}\text { trans-Linalool } \\
\text { oxide }\end{array}$ & 1088 & 1090 & - & - & 0.09 & - & - & - & 0.57 & - & - & - & - \\
\hline 21 & $\alpha$-Terpinolene & 1088 & 1091 & - & - & - & 0.07 & - & - & - & - & 0.25 & - & - \\
\hline 22 & $n$-Undecane & 1100 & 1099 & 0.63 & 0.98 & 1.01 & 0.65 & 1.24 & 0.81 & 1.47 & 0.49 & 1.34 & 1.37 & 1.03 \\
\hline 23 & Nonanal & 1098 & 1103 & 0.09 & 0.08 & 0.08 & 0.05 & 0.04 & 0.07 & - & 0.04 & 0.13 & - & - \\
\hline 24 & cis-Thujone & 1101 & 1111 & - & - & - & - & - & - & - & - & - & 0.13 & 0.14 \\
\hline 25 & endo-Fenchol & 1121 & 1127 & - & - & - & - & 0.07 & - & 0.04 & - & - & 0.41 & 0.04 \\
\hline 26 & $\alpha$-Campholenal & 1125 & 1130 & 0.19 & 0.26 & 0.26 & 0.05 & 0.49 & 0.28 & 0.75 & 0.07 & 0.46 & 3.17 & 1.14 \\
\hline 27 & $\begin{array}{l}\text { trans- } \\
\text { Pinocarveol }\end{array}$ & 1135 & 1139 & 0.16 & 0.18 & 0.20 & 0.07 & 0.27 & 0.14 & 0.37 & 0.09 & - & 1.72 & - \\
\hline 28 & cis-Verbenol & 1137 & 1142 & - & - & - & - & - & - & - & - & - & - & 0.13 \\
\hline 29 & trans-Verbenol & 1140 & 1145 & 0.27 & - & 0.38 & 0.07 & 0.39 & - & 0.40 & 0.12 & - & 2.17 & - \\
\hline 30 & Camphor & 1141 & 1149 & - & - & - & - & - & - & - & - & - & - & 1.10 \\
\hline 31 & $\begin{array}{l}\text { trans- } \\
\text { Pinocamphone }\end{array}$ & 1160 & 1166 & - & - & - & - & - & - & - & - & - & - & 0.04 \\
\hline 32 & Pinocarvone & 1162 & 1167 & tr. & - & - & - & 0.07 & - & 0.09 & - & - & - & 0.09 \\
\hline 33 & Borneol & 1165 & 1171 & 0.34 & 0.32 & 0.30 & - & - & - & 0.33 & - & - & - & - \\
\hline 34 & $\begin{array}{l}p \text {-Mentha-1,5- } \\
\text { dien-8-ol }\end{array}$ & 1172 & 1177 & - & - & - & - & - & - & - & 0.13 & - & 1.82 & 0.64 \\
\hline 35 & Terpinene-4-ol & 1177 & 1181 & - & 0.09 & 0.10 & 0.06 & 0.12 & 0.09 & 0.07 & 0.08 & - & 0.21 & 0.11 \\
\hline 36 & $p$-Cymene-8-ol & 1183 & 1187 & - & - & 0.09 & 0.05 & - & - & 0.08 & - & - & 0.12 & 0.15 \\
\hline 37 & $\alpha$-Terpineol & 1189 & 1196 & 0.15 & 0.23 & 0.20 & 0.08 & 0.27 & 0.10 & 0.17 & 0.07 & 0.19 & 0.74 & 0.21 \\
\hline 38 & Myrtenol & 1194 & 1200 & 0.11 & 0.14 & 0.16 & 0.06 & 0.20 & 0.11 & 0.19 & 0.07 & - & - & 0.31 \\
\hline 39 & $\begin{array}{l}\text { Methyl } \\
\text { chavicol }\end{array}$ & 1195 & 1201 & - & - & - & - & - & - & - & - & 0.40 & - & - \\
\hline 40 & Myrtenal & 1195 & 1202 & - & - & - & - & - & - & - & - & - & 0.51 & - \\
\hline 41 & Verbenone & 1204 & 1207 & 0.23 & 0.22 & 0.31 & 0.07 & 0.28 & 0.11 & 0.20 & 0.13 & 0.33 & 2.41 & 0.57 \\
\hline 42 & trans-Carveol & 1215 & 1219 & 0.12 & 0.13 & 0.11 & 0.02 & 0.16 & 0.13 & 0.19 & 0.05 & 0.17 & 1.12 & 0.49 \\
\hline 43 & $\begin{array}{l}\text { trans-Chrys- } \\
\text { anthemyl } \\
\text { acetate }\end{array}$ & 1235 & 1240 & - & 0.09 & 0.12 & - & - & - & - & - & - & - & - \\
\hline 44 & $\begin{array}{l}\text { Carvacrol } \\
\text { methyl ether }\end{array}$ & 1240 & 1246 & - & - & - & - & - & - & - & - & - & - & 0.24 \\
\hline 45 & Carvone & 1243 & 1247 & - & - & - & - & - & - & - & - & - & 0.12 & - \\
\hline
\end{tabular}


T a ble 2 (cont.)

\begin{tabular}{|c|c|c|c|c|c|c|c|c|c|c|c|c|c|c|}
\hline No. & Components & $\mathbf{K I L}^{\mathbf{a})}$ & $\mathbf{K I E}^{\mathbf{b})}$ & HP01 & HP02 & HP03 & HP04 & HP05 & HP06 & HP07 & HP08 & HP09 & HP10 & HP11 \\
\hline 46 & Carvotanacetone & 1246 & 1251 & - & - & - & - & - & - & - & - & - & 0.15 & 0.05 \\
\hline 47 & Gearniol & 1249 & 1255 & - & 0.17 & 0.16 & 0.05 & - & - & 0.17 & - & 0.21 & - & - \\
\hline 48 & cis-Geraniol & 1255 & 1259 & - & - & - & - & 0.14 & - & - & - & - & - & - \\
\hline 49 & $\begin{array}{l}\text { 2-Methyl } \\
\text { dodecane }\end{array}$ & 1268 & 1273 & 1.12 & 2.06 & 2.24 & 0.78 & 1.26 & - & 1.51 & - & 2.11 & 1.30 & 0.85 \\
\hline 50 & E-Anethole & 1282 & 1286 & - & - & - & - & - & - & - & - & 5.79 & 0.54 & 0.43 \\
\hline 51 & Bornyl acetate & 1285 & 1291 & - & - & 0.10 & 0.06 & 0.05 & 0.06 & 0.06 & 0.07 & - & - & - \\
\hline 52 & Thymol & 1290 & 1299 & 0.13 & 0.15 & 0.12 & 0.06 & 0.11 & 0.08 & 0.05 & 0.08 & - & 0.22 & 1.43 \\
\hline 53 & Tridecane & 1294 & 1301 & 0.25 & 0.51 & 0.58 & 0.23 & 0.25 & 0.19 & 0.33 & 0.24 & 0.55 & 0.29 & - \\
\hline 54 & Carvacrol & 1298 & 1353 & 0.68 & 0.30 & 0.28 & 0.10 & 0.31 & 0.22 & 0.21 & 0.14 & 0.32 & 0.87 & 5.60 \\
\hline 55 & $\alpha$-Longipinene & 1350 & I & - & 0.34 & 0.56 & - & - & - & - & - & 0.37 & 0.54 & - \\
\hline 56 & $\alpha$-Cubebene & 1351 & I & - & - & - & 0.19 & 0.05 & 0.11 & 0.06 & 0.18 & - & - & - \\
\hline 57 & $\alpha$-Ylangene & 1373 & 1381 & - & 0.31 & 0.38 & 0.24 & 0.22 & 0.18 & 0.19 & 0.17 & 0.28 & 0.08 & 0.09 \\
\hline 58 & $\alpha$-Copaene & 1376 & 1389 & 0.37 & 0.52 & 0.66 & 0.46 & 0.45 & 0.42 & 0.55 & 0.28 & 0.61 & 0.21 & 0.52 \\
\hline 59 & $\beta$-Bourbonene & 1384 & 1397 & 0.14 & 0.16 & 0.18 & 0.16 & 0.17 & 0.24 & 0.17 & 0.18 & 0.35 & - & - \\
\hline 60 & $\beta$-Elemene & 1391 & 1410 & tr. & - & - & - & - & 0.46 & 0.11 & 0.63 & - & - & 0.20 \\
\hline 61 & Italicene & 1405 & 1415 & - & 0.11 & 0.16 & - & - & - & - & - & - & - & - \\
\hline 62 & $\begin{array}{l}\text { 2-epi- } \beta \text { - } \\
\text { Funebrene }\end{array}$ & 1411 & 1422 & 0.26 & 0.24 & - & - & - & - & - & - & - & - & - \\
\hline 63 & $\beta$-Cedrene & 1418 & 1424 & - & - & - & - & 0.18 & - & - & - & - & - & - \\
\hline 64 & $\begin{array}{l}\text { trans }-(E)- \\
\text { Caryophyllene }\end{array}$ & 1418 & 1432 & 6.22 & 7.61 & 4.88 & 19.27 & 4.40 & 10.24 & 4.24 & 11.69 & 5.74 & 0.50 & 1.22 \\
\hline 65 & $\beta$-Copaene & 1430 & 1446 & 0.19 & 0.34 & 0.46 & - & 0.20 & - & 0.16 & - & 0.20 & - & - \\
\hline 66 & Aromadendrene & 1439 & 1452 & 0.11 & 0.25 & 0.38 & 0.30 & 0.17 & 0.11 & 0.09 & 0.23 & 0.23 & - & 0.11 \\
\hline 67 & $\alpha$-Himachalene & 1449 & 1455 & 0.16 & 0.17 & - & - & - & - & - & - & 0.18 & 0.26 & - \\
\hline 68 & $\alpha$-Hummulene & 1452 & 1460 & - & - & - & - & - & 1.88 & - & - & - & - & - \\
\hline 69 & $\beta$-(Z)-Farnesene & 1454 & 1465 & 2.45 & 2.98 & 3.76 & 2.47 & 1.67 & - & 1.01 & 4.40 & 2.39 & 0.19 & 0.94 \\
\hline 70 & Sesquisabinene & 1457 & 1471 & - & - & - & 2.48 & - & - & - & - & - & - & - \\
\hline 71 & $\begin{array}{l}\text { 4,5-di-epi- } \\
\text { Aristolochene }\end{array}$ & 1467 & 1476 & - & - & - & - & - & - & - & 0.86 & - & - & 0.52 \\
\hline 72 & Dodecanol & 1473 & 1482 & 5.22 & 1.71 & 1.55 & 6.69 & & 4.76 & 2.00 & - & - & - & 1.02 \\
\hline 73 & $\gamma$-Muurolene & 1477 & 1486 & - & - & 2.20 & 1.45 & 2.82 & - & - & 4.05 & 1.49 & 0.29 & 0.42 \\
\hline 74 & Germacrene D & 1480 & 1489 & - & 1.82 & 1.05 & - & - & - & - & - & 0.76 & - & - \\
\hline 75 & $\beta$-Selinene & 1485 & 1499 & 7.26 & 3.21 & 1.34 & 6.10 & 7.05 & 11.38 & 6.42 & 13.86 & 1.87 & 3.73 & 10.26 \\
\hline 76 & $\alpha$-Selinene & 1494 & 1501 & 6.16 & 3.61 & 1.93 & 7.01 & 6.15 & 9.89 & 4.48 & 10.63 & 2.04 & 0.41 & 5.99 \\
\hline 77 & $\alpha$-Muurolene & 1495 & 1512 & - & - & 0.35 & - & - & - & - & - & - & - & - \\
\hline 78 & $\alpha$-Farnesene & 1505 & 1516 & - & - & 0.23 & - & - & - & - & - & - & - & - \\
\hline 79 & $\beta$-Bisabolene & 1505 & 1525 & 0.27 & - & - & & - & - & - & - & - & - & - \\
\hline 80 & $\gamma$-Cadinene & 1513 & 1528 & 0.51 & 0.78 & 1.06 & 1.08 & 0.61 & 0.60 & 0.44 & 0.87 & 0.74 & 0.18 & 0.38 \\
\hline 81 & 7-epi- $\alpha$-Selinene & 1520 & 1537 & 0.21 & 0.10 & - & - & 0.25 & 0.36 & 0.17 & 0.46 & - & - & 0.26 \\
\hline 82 & $\delta$-Cadinene & 1524 & 1541 & 1.10 & 1.51 & 2.12 & 2.03 & 1.18 & 1.13 & 0.90 & 1.56 & 1.44 & 0.18 & 0.70 \\
\hline 83 & $\begin{array}{l}\text { trans-Cadina- } \\
1,4 \text {-diene }\end{array}$ & 1532 & 1551 & - & 0.38 & 0.21 & 0.26 & 0.16 & 0.17 & 0.12 & 0.29 & 0.18 & - & - \\
\hline 84 & $\alpha$-Cadinene & 1538 & 1569 & - & - & 0.37 & 0.31 & 0.24 & 0.22 & 0.18 & 0.28 & 0.27 & - & 0.70 \\
\hline 85 & $\alpha$-Calacorene & 1547 & 1573 & 0.20 & 0.26 & 0.34 & 0.26 & 0.36 & 0.17 & 0.15 & 0.26 & 0.25 & 0.10 & 0.22 \\
\hline 86 & $E$-Nerolidol & 1564 & 1580 & 1.04 & 1.08 & 1.59 & 0.83 & 0.63 & 0.88 & 0.56 & 1.26 & 1.03 & 0.32 & 0.42 \\
\hline 87 & Dodecanoic acid & 1568 & 1584 & - & - & - & - & - & - & - & - & - & 0.32 & - \\
\hline 88 & Spathulenol & 1576 & 1594 & - & 1.56 & 1.62 & 6.29 & 0.16 & - & 3.30 & 5.18 & 1.75 & 0.39 & 1.25 \\
\hline 89 & $\begin{array}{l}\text { Caryophyllene } \\
\text { oxide }\end{array}$ & 1581 & 1596 & 11.32 & 5.38 & 4.17 & 3.50 & 4.32 & 12.35 & 3.01 & 2.93 & 5.11 & 1.15 & 1.70 \\
\hline 90 & Viridiflorol & 1590 & 1607 & - & - & 0.54 & - & - & - & - & - & - & - & - \\
\hline 91 & Longiborneol & 1592 & 1611 & - & - & 0.38 & - & - & - & - & - & - & - & - \\
\hline 92 & Ledol & 1602 & 1622 & - & 0.40 & - & - & 0.41 & - & - & 0.87 & - & - & - \\
\hline 93 & $\begin{array}{l}\text { Humulene } \\
\text { epoxide II }\end{array}$ & 1606 & 1631 & - & 0.49 & 0.87 & - & - & - & 0.38 & - & - & - & - \\
\hline
\end{tabular}


T a b l e 2 (cont.)

\begin{tabular}{|c|c|c|c|c|c|c|c|c|c|c|c|c|c|c|}
\hline No. & Components & KIL $^{\text {a) }}$ & $\mathbf{K I E}^{\mathbf{b})}$ & HP01 & HP02 & HP03 & HP04 & HP05 & HP06 & HP07 & HP08 & HP09 & HP10 & HP11 \\
\hline 94 & $\begin{array}{l}\text { 1,10-di-epi- } \\
\text { Cubenol }\end{array}$ & 1618 & 1640 & - & - & - & - & - & - & - & - & 0.33 & - & - \\
\hline 95 & 1-epi-Cubenol & 1627 & 1645 & - & - & 0.40 & 0.24 & - & - & 0.22 & 0.35 & - & - & - \\
\hline 96 & $\begin{array}{l}\text { cis-Cadin-4-en- } \\
7 \text {-ol } \\
\text { epi- } \alpha-\end{array}$ & 1635 & 1649 & - & - & - & - & - & - & - & - & - & 0.51 & - \\
\hline 97 & $\begin{array}{l}\text { Murrolol+epi- } \alpha- \\
\text { Cadinol }\end{array}$ & 1641 & 1655 & - & - & 5.16 & - & - & - & - & - & - & - & - \\
\hline 98 & $\begin{array}{l}\alpha \text {-Muurolol } \\
\text { (Torreyol) }\end{array}$ & 1645 & 1659 & - & 1.94 & - & - & - & - & - & - & - & - & - \\
\hline 99 & Vulgarone B & 1649 & 1661 & - & - & 0.37 & - & - & - & - & - & - & 0.14 & - \\
\hline 100 & $\alpha$-Cadinol & 1653 & 1675 & - & - & 0.70 & 1.79 & - & - & - & 2.96 & 0.65 & - & - \\
\hline 101 & neo-Intermedeol & 1656 & 1681 & 2.51 & - & - & - & 1.63 & 2.38 & 1.59 & - & - & - & 1.68 \\
\hline 102 & $n$-Tetradecanol & 1671 & 1730 & 7.63 & 7.10 & - & - & 1.83 & - & 2.57 & 2.28 & - & 1.60 & - \\
\hline 103 & $\alpha$-Cadalene & 1675 & 1764 & - & - & 7.77 & - & 4.35 & - & - & - & - & - & - \\
\hline 104 & $\begin{array}{l}\text { Amorpha-4,9- } \\
\text { dien-2-ol }\end{array}$ & 1700 & 1765 & - & - & - & - & - & - & 3.00 & - & - & - & - \\
\hline 105 & $\begin{array}{l}\text { Methyl } \\
\text { tetradecanoate }\end{array}$ & 1726 & 1778 & 0.25 & 0.22 & 0.32 & - & 0.33 & 0.25 & 0.21 & 0.33 & 0.42 & 0.07 & 0.18 \\
\hline 106 & Cyclocolorenone & 1759 & 1780 & - & 0.10 & - & - & - & - & - & 0.23 & - & - & - \\
\hline 107 & Benzyl benzoate & 1759 & 1839 & 0.23 & 0.15 & 0.32 & - & - & 0.16 & 0.14 & 0.37 & 0.37 & - & 0.21 \\
\hline 108 & $n$-Pentadecanol & 1773 & 1854 & - & - & - & 6.92 & 0.11 & - & - & - & - & 0.07 & - \\
\hline 109 & $\begin{array}{l}\text { epi- } \\
\text { Cyclocolorenone }\end{array}$ & 1774 & 1886 & - & - & - & - & 0.15 & - & - & - & - & - & - \\
\hline 110 & 2-Pentadecanone & 1835 & 1888 & 0.72 & - & 1.66 & - & - & 0.64 & 0.53 & 0.86 & 1.09 & - & 0.43 \\
\hline 111 & $\begin{array}{l}\text { 6,10,14-trimethyl } \\
\text { 2-Pentadecanone }\end{array}$ & 1848 & 1899 & - & 1.32 & - & 0.81 & 0.67 & - & - & - & - & 0.43 & - \\
\hline 112 & $n$-Hexadecanol & 1879 & 1933 & 0.85 & 2.48 & 1.04 & 1.73 & - & 1.05 & 0.88 & - & 0.71 & 0.23 & 0.15 \\
\hline 113 & Cyclohexadecane & 1881 & 1975 & - & - & - & - & 2.24 & - & - & 1.78 & - & - & - \\
\hline 114 & Nonadecane & 1900 & 2001 & 0.39 & 0.61 & 0.91 & 0.23 & 0.17 & 0.18 & 0.18 & 0.35 & 0.68 & 0.12 & 0.15 \\
\hline 115 & $\begin{array}{l}\text { Methyl } \\
\text { hexadecanoate }\end{array}$ & 1927 & 2062 & 0.38 & 0.70 & 0.69 & 0.54 & - & 0.53 & 0.49 & - & 0.70 & - & 0.29 \\
\hline 116 & $\begin{array}{l}\text { Hexadecanoic } \\
\text { acid }\end{array}$ & 1970 & 2099 & 0.55 & 0.11 & 0.96 & 0.52 & 0.81 & 0.53 & 0.41 & 1.29 & 0.48 & 0.22 & 0.28 \\
\hline 117 & Eicosane & 2000 & 2101 & 0.17 & 0.27 & 0.26 & 0.09 & 0.09 & 0.09 & 0.09 & 0.20 & 0.23 & 0.04 & 0.06 \\
\hline 118 & Manool & 2056 & 2129 & - & - & - & - & - & - & - & - & - & - & 0.07 \\
\hline 119 & Methyl linoleate & 2095 & 2145 & - & 0.20 & 0.19 & 0.18 & 0.24 & 0.12 & - & 0.13 & 0.18 & 0.07 & 0.06 \\
\hline 120 & Heneicosane & 2100 & 2199 & 0.99 & 2.18 & 2.16 & 0.94 & 0.97 & 0.76 & 0.84 & 1.36 & 1.58 & 0.44 & 0.60 \\
\hline 121 & $\begin{array}{l}\text { Methyl } \\
\text { octadecanoate }\end{array}$ & 2124 & 2300 & - & - & - & 0.04 & 0.06 & - & - & 0.04 & - & - & - \\
\hline 122 & $\begin{array}{l}\text { (Z)-9-Octa- } \\
\text { decenoic acid }\end{array}$ & 2137 & 2401 & - & - & - & - & - & - & - & 0.12 & - & - & - \\
\hline 123 & Docosane & 2200 & 2501 & 0.10 & 0.20 & 0.26 & 0.09 & 0.07 & 0.07 & 0.08 & 0.10 & 0.20 & 0.05 & 0.05 \\
\hline 124 & Tricosane & 2300 & 1730 & 0.24 & 0.59 & 0.75 & 0.27 & 0.23 & 0.21 & 0.25 & 0.29 & 0.55 & 0.38 & 0.28 \\
\hline 125 & Tetracosane & 2400 & 1764 & - & - & 0.08 & 0.03 & - & - & 0.02 & 0.03 & - & - & - \\
\hline 126 & Pentacosane & 2500 & 1765 & - & 0.37 & 0.22 & 0.22 & 0.08 & - & 0.03 & 0.16 & 0.14 & 0.04 & 0.30 \\
\hline
\end{tabular}

\begin{tabular}{|c|c|c|c|c|c|c|c|c|c|c|c|}
\hline Chemical classes & HP01 & HP02 & HP03 & HP04 & HP05 & HP06 & HP07 & HP08 & HP09 & HP10 & HP11 \\
\hline $\begin{array}{l}\text { Monoterpene } \\
\text { hydrocarbons (MH) }\end{array}$ & 15.75 & 20.54 & 17.60 & 3.09 & 30.21 & 8.72 & 34.70 & 1.94 & 24.39 & 47.21 & 30.77 \\
\hline $\begin{array}{l}\text { Oxygen-containing } \\
\text { monoterpenes }(\mathrm{OM})\end{array}$ & 2.51 & 2.45 & 3.02 & 0.80 & 2.93 & 1.41 & 3.94 & 1.14 & 1.68 & 15.89 & 12.77 \\
\hline $\begin{array}{l}\text { Sesquiterpene } \\
\text { hydrocarbons (SH) }\end{array}$ & 25.61 & 24.70 & 22.62 & 44.07 & 26.33 & 37.56 & 19.44 & 50.88 & 19.39 & 6.67 & 22.53 \\
\hline $\begin{array}{l}\text { Oxygen-containing } \\
\text { sesquiterpenes (OS) }\end{array}$ & 14.87 & 10.95 & 15.80 & 12.65 & 7.30 & 15.61 & 12.06 & 13.78 & 8.87 & 2.51 & 5.05 \\
\hline Diterpenes (D) & 0.00 & 0.00 & 0.00 & 0.00 & 0.00 & 0.00 & 0.00 & 0.00 & 0.00 & 0.00 & 0.07 \\
\hline $\begin{array}{l}\text { Non-terpene } \\
\text { components (NT) }\end{array}$ & 24.32 & 26.87 & 29.25 & 24.73 & 19.03 & 14.05 & 17.68 & 12.28 & 23.04 & 13.66 & 11.68 \\
\hline Total $(\%)$ & 83.06 & 85.51 & 88.29 & 85.34 & 85.80 & 77.35 & 87.82 & 80.02 & 77.37 & 85.94 & 82.87 \\
\hline
\end{tabular}

a) KIL - Kovat's Index Literature [27]; b) KIE - Kovat's Index Estimated (AMDIS) [28]; tr. = traces $(<0.02 \%)$. 
In several Lithuanian HP samples, the sesquiterpene hydrocarbons and oxygenated sesquiterpenes made up $62.00-81.80 \%$ of the EO. The aliphatic compounds (2-methyl octane, $n$-nonane, $n$ decane, $n$-undecane, $n$-tetradecanol, etc.) varied from 1.70 to $19.60 \%$ [29]. Terpenoid constituents, particularly sesquiterpenes, were described as primary components in Serbian HP EOs [30]. In Sardinia the sesquiterpene hydrocarbons had the highest contribution $(36.80 \%)$. This fraction was dominated by germacrene D $(17.60 \%)$. The monoterpene hydrocarbons represented $28.40 \%$ of the total EO with $\alpha$-pinene $(15.80 \%)$ as the major compound. The oxygenated sesquiterpenoid fraction was relatively poor. It represented $4.60 \%$ of the total EO with cis-cadin-4-en-7-ol (1.70\%) as the major constituent. The oxygenated monoterpenes constituted only $1.00 \%$. Compared to these results, our tested EOs contained $6.67-50.88 \%$ of sesquiterpene hydrocarbons with trans- $(E)$-caryophyllene $(0.50-19.27 \%)$, and $\alpha$ - and $\beta$-selinene (0.41-10.63\% and $1.34-13.86 \%$, respectively) as the dominant components and $1.94-47.21 \%$ of monoterpene hydrocarbons with $\alpha$-pinene as the dominant component (1.15-36.74\%). Furthermore, the content of oxygen-containing derivatives, both mono- and sesquiterpenes, was the opposite, as our tested samples comprised $0.80-15.89 \%$ of oxygencontaining mono- and $2.51-15.80 \%$ of oxygencontaining sesquiterpenes.

GC/FID/MS analyses of the isolated EOs revealed a total of 126 compounds representing $77.35-88.29 \%$ of the oils (Table 2). Twenty five components were detected in all analyzed EOs and seven of these compounds were found in amounts higher than $3.00 \%$. These components were considered principal components of HP EOs: $\alpha$-pinene (2.03-36.74\%), $\beta$-pinene $(0.36-6.89 \%)$, 2-methyldecane $(0.82-3.14 \%)$, carvacrol $(0.14-5.60 \%)$, trans- $(E)$-caryophyllene $(0.5-19.27 \%), \beta$-selinene $(1.34-13.86 \%)$ and caryophyllene oxide (1.15$12.35 \%)$. Although chemical diversity of the HP EOs was high, two main types of HP EOs can generally be distinguished: pinene-type (populations HP02, HP03, HP05, HP07, HP09, HP10 and HP11 with EO reach in $\alpha$-pinene) and caryophyllene-type (populations HP01, HP04, HP06 and HP08 with EO rich in trans- $(E)$-caryophyllene and caryophyllene oxide), which was confirmed by PCA analysis (Fig. 1).

Concerning the dominant components in the EOs from HP growing wild in Lithuania, three different chemotypes (trans-(E)-caryophyllene, caryophyllene oxide and germacrene $\mathrm{D})$ have been reported [32].

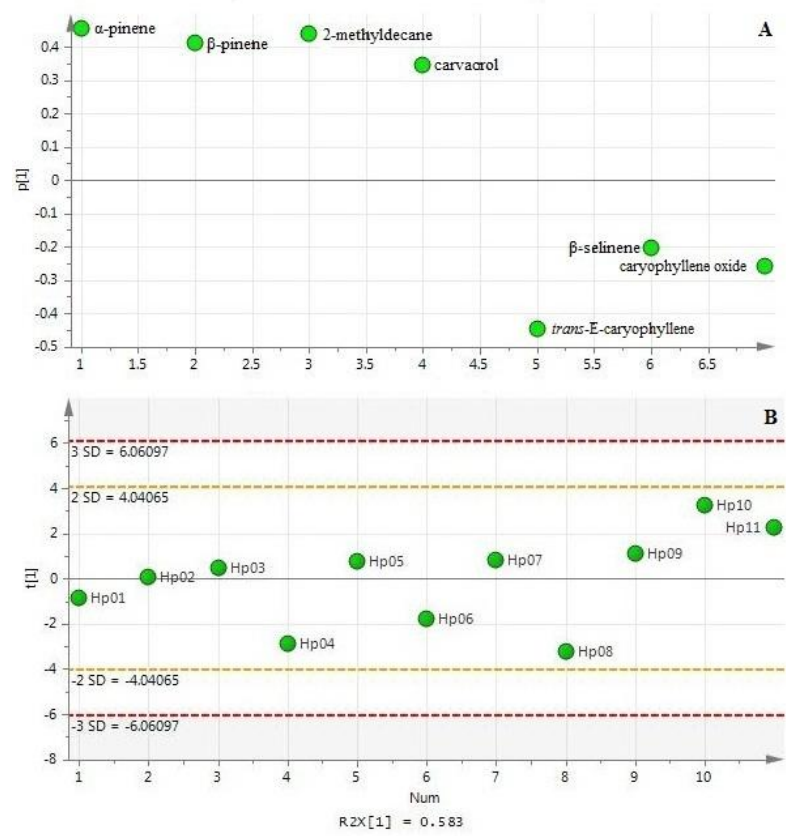

Fig. 1. PCA analysis of the seven main EO components and their influence (A) on the distinguishing two types of populations (pinene-type, above baseline and caryophyllene-type, below baseline) (B)

In general, the components $\beta$-pinene, $\alpha$ pinene, 2-methyloctane, spathulenol, caryophyllene oxide, germacrene $\mathrm{D}$ and trans- $(E)$-caryophyllene were the most frequently reported constituents in Hypericum EOs [33]. For instance, the EO of HP from southeastern France was rich in $\alpha$ pinene and the EO of HP ssp. angustifolium, also from France, was characterized by spathulenol $(21.10 \%)$ and tetradecanol $(9.10 \%)$ [19, 20, 34]. On the other hand, 2-methyloctane (21.10\%), germacrene-D (17.60\%) and $\alpha$-pinene $(15.80 \%)$ were the major compounds in the same subspecies in Italy [35].

Germacrene D was the main component found in the hydrodistilled EOs from wild-grown $(22.80 \%)$ and cultivated plants $(16.90 \%)$ from several regions of Greece, followed by 2-methyloctane $(10.80-17.80 \%)$, trans- $(E)$-caryophyllene $(6.60-10.30 \%), \quad \alpha$-pinene $(5.20-10.10 \%)$ and bicyclogermacrene $(4.10-4.80 \%)$ [36]. In the same way, $\alpha$ - and $\beta$-pinene were found to be the main components in another study of Greek HP [37].

HP collected from the Barelić region in Serbia contained $8.60 \%$ of $\alpha$-pinene, but it was absent from plant material from the Rujan region [30]. In the same study, trans-(E)-caryophyllene and caryophyllene oxide were reported to be principal components. In the region of Kosovo, HP were characterized by the following main constituents: $\alpha$-pinene (3.70-36.50\%), 2-methyl-octane (1.10$15.50 \%)$, trans- $(E)$-caryophyllene $(1.20-12.40 \%)$, 
caryophyllene oxide (3.30-17.70\%) and $n$-tetradecanol (3.60-10.40\%) [31].

According to these data, our EOs have the largest similarity to the HP EOs from the neighboring regions of Greece, Serbia and Kosovo, as $\alpha$-pinene, trans- $(E)$-caryophyllene and caryophyllene oxide were found to be the predominant components.

According to some authors, qualitative and quantitative variation in the content of secondary metabolites in HP is influenced by genetic, ecological and environmental factors [38, 39]; thus, it is very difficult to compare data about $\mathrm{EO}$ with scarce information on the status of plant material (fresh/dry) used for hydrodistillation, as well as the plant development status or the environmental variables of collection sites. Also, EOs from HP wild populations and cultivated in uniform conditions are very likely to be genetically determined because no considerable differences were found in the composition of the volatile constituents [18]. However, obtained information about EO yield and composition of the studied Albanian HP can help to assess the potential of these populations for further sustainable wild exploitation and can also be taken into a consideration as a resource of valuable genetic material for further cultivation and breeding.

\section{REFERENCES}

[1] N. K. B. Robson, Hypericum Botany, Hypericum: The Genus Hypericum. Taylor and Francis, New York, USA, 2003.

[2] European Pharmacopoeia, 6th Ed. Council of Europe, Strasbourg, France, 2008.

[3] R. Berghöfer, Analytik und Isolierung phenolischer Inhaltsstoffe von Hypericum perforatum L. aus Anbau und Wildvorkommen und Vergleich mit anderen heimischen Hypericum-Arten. Cramer, Stuttgart, Berlin, Germany, 1987.

[4] K. Linde, G. Ramirez, C. D. Mulrow, A. Pauls, W. Weidenhammer, D. Melchart, St. John's wort for depression: an overview and meta-analysis of randomized clinical trials. Br. Med. J., 313, 253-258 (1996).

[5] S. L. Crockett, N. K. B. Robson, Taxonomy and chemotaxonomy of the genus Hypericum. Medicinal and Aromatic Plant Sci. Biotech. (in press).

[6] W. E. Müller, St. John's wort and its active principles in depression and anxiety, Birkhäuser Verlag, Basel, Switzerland, 2005.

[7] American Botanical Council, St. John's Wort. The Complete German Commission E monographs: Therapeutic guide to herbal medicines, Austin, Texas, 1998, pp. 214-215.
[8] European Medicines Agency, Assessment report on Hypericum perforatum L., herba, London, UK, 2008.

[9] Commission Suisse de Pharmacopée. Pharmacopoea Helvetica. 8th ed. Département fédéral de l'intérieur; Berne, Switzerland, 1997.

[10] J. Reichling, A. Weselerand, R. Saller, A current review of the antimicrobial activity of Hypericum perforatum L., Pharmacopsychiatry, 34, 116-118 (2001).

[11] S. Kasper, Hypericum perforatum-A review of clinical studies, Pharmacopsychiatr.y, 34, 51-55 (2001).

[12] I. Lopez-Bazzocchi, J. B. Hudson, G. H. Towers, Antiviral activity of the photoactive plant pigment hypericin, Photochem. Photobiol., 54, 95-98 (1991).

[13] D. Meruelo, G. Lavie, D. Lavie, Therapeutic agents with dramatic antiretroviral activity and little toxicity at effective doses: aromatic polycyclic diones hypericin and pseudohypericin, Proc. Natl. Acad. Sci. USA, 85, 5230-5234 (1988).

[14] P. Miskovsky, Hypericin - a new antiviral and antitumor photosensitizer: mechanism of action and interaction with biological macromolecules, Curr. Drug Targets, 3, 55-84 (2002).

[15] A. I. Gurevich, V. N. Dobrynin, M. N. Kolosov, S. A. Popravko, I. D. Riabova, Antibiotic hyperforin from Hypericum perforatum L., Antibiotiki, 16, 510-513 (1971).

[16] P. Maisenbacher, K. A. Kovar, Analysis and stability of Hyperici oleum, Planta Med., 58, 351354 (1992).

[17] R. Melzer, U. Fricke, J. Holzl, Vasoactive properties of procyanidins from Hypericum perforatum L. in isolated porcine coronary arteries, Arzneimittel-Forschung, 41, 481-483 (1991).

[18] P. A. De Smet, W. A. Nolen, St John's wort as an antidepressant, Br. Med. J., 313, 253-258 (1996).

[19] I. Schwob, J. M. Bessiere, J. Viano, Composition of the essential oils of Hypericum perforatum $\mathrm{L}$. from southeastern France, C. R. Biologies, 325, 781-785 (2002).

[20] C. Mathis, G. Ourisson, Chemotaxonomic study of the genus Hypericum. IV. Distribution of sesquiterpenes, monoterpene alcohols, and saturated aldehydes in the essential oils of Hypericum. Phytochemistry, 3, 377-378 (1964).

[21] A. Nahrstedt, V. Butterweck, Biologically active and other chemical constituents of the herb of Hypericum perforatum L., Pharmacopsychiatry, 30 (Suppl.), 129-134 (1997).

[22] L. Stjepanović, M. Ćorović, R. Nikolić, S. Palović, P. Živanović, Essential oil content and number of oil vesicles in Hypericum species from various 
habitats of Tara mountain, Arhiv za farmaciju, 15, 177-188 (1965).

[23] L. Roth, Hypericum, hypericin: Botanik, Inhaltstoffe, Wirkung. Ecomed Verlagsgesellschaft GmbH, Landsberg, Germany, 1990.

[24] R. Omidbaigi, M. Azizi, Effect of time of harvest on hypericin and essential oil content of Hypericum perforatum L. from Iran, Iran Agric. Res., 19, 155-164 (2000).

[25] R. Lutz, The essential oils of different St. John's worts, Ätherische Öle, Riechstoffe, Parfümerien Essenzen und Aromen, 2, 137-139 (1952).

[26] Personal communications: Aura Cacia (Urbana, Iowa, USA) and Mountain Rose Herbs (Eugene, Oregon, USA).

[27] R. P. Adams, Identification of Essential Oil Components by Gas Chromatography/Mass Spectrometry, Allured Publishing Corporation, IL, USA, 2007.

[28] Automated Mass Spectral Deconvolution and Identification System software (AMDIS ver.2.1.), National Institute of Standards and Technology (NIST), Standard Reference Data Program, Gaithersburg, MD (USA).

[29] SIMCA $13^{\circledR}$, Umetrics AB, Sweden.

[30] B. Gudzić, S. Đordević, R. Palić, G. Stojanović, Essential oils Hypericum olympicum L. and Hypericum perforatum L., Flavour Frag. J., 16, 201-203 (2001).

[31] A. Hajdari, B. Mustafa, D. Nebija, A. Kashtanjeva, J. Widelski, K. Glowniak, J. Novak, Essential oil composition and variability of Hypericum perforatum L. from wild population in Kosovo, Curr. Issues Pharm. Med. Sci., 27, 51-54 (2014).
[32] D. Mockute, G. Bernotiene, A. Judzentiene, Volatile compounds of the aerial parts of wild St. John's wort (Hypericum perforatum L.) plants, Chemija (Vilnius), 14, 108-111 (2003).

[33] S. L. Crockett, Essential Oil and Volatile Components of the Genus Hypericum (Hypericaceae), Nat. Prod. Commun., 5, 1493-1506 (2010).

[34] I. Schindler, J.-M. Bessiere, J. Viano, Composition of the essential oils of Hypericum perforatum $\mathrm{L}$. from southeastern France, C. R. boil., 325, 781785 (2002).

[35] G. Pintore, M. Chessa, G. Boatto, R. Cerri, M. Usai, B. Tirillini, Essential oil composition of Hypericum perforatum L. var. angustifolium DC Growing Wild in Sardinia (Italy), J. Essent. Oil Res., 17, 533-535 (2005).

[36] P. S. Chatzopoulou, T. V. Koutsos, S. T. Katsiotis, Chemical Composition of the Essential Oils from Cultivated and Wild Grown St. John's wort (Hypericum perforatum), J. Essent. Oil Res., 2006, 18, 643 (2006)

[37] P. V. Petrakis, M. Couladis, V. Roussis, A method for detecting the biosystematic significance of the essential oil composition: The case of five Hellenic Hypericum L. species, Biochem. Syst. Ecol., 33, 873-898 (2005).

[38] E. Miraldi, M. Biagi, D. Giachetti, Chemical constituents and effects of topical application of Oleum Hyperici on skin sensitivity to stimulated sun exposure, Nat. Prod. Commun., 1, 209-213 (2006).

[39] G. Zdunić, D. Godevac, M. Milenković, D. Vucićević, K. Savikin, N. Menkovic, S. Petrovic, Evaluation of Hypericum perforatum oil extracts for an anti-inflammatory and gastroprotective activity in rats, Phytother. Res., 23, 1559-1564 (2009). 